[Article]

\title{
二元混合离子液体的电导率与离子间的缔合作用
}

\author{
宁汇侯民强杨德重康欣晨 韩布兴
}

(中国科学院化学研究所, 中国科学院胶体界面与化学热力学重点实验室, 北京分子科学国家实验室, 北京 100190)

\begin{abstract}
摘要: 在 293.15-323.15 K 范围内, 测定了 13 种常见离子液体及其 25 组混合体系的电导率. 利用 Vogel-Tammann-Fulcher (VTF) 方程对电导率数据进行拟合, 并通过方程式中的拟合参数分析了离子液体混合 后其阴阳离子间缔合作用的变化规律. 结果表明, 在相同温度下, 离子液体的阳离子侧链越短, 阴离子电荷越分 散, 阴阳离子间的氢键作用力越弱, 离子液体的电导率越大, 其中阴离子的影响比阳离子更明显. 混合离子液体 中离子间的缔合作用不仅与阴阳离子的种类有关, 而且与混合物的组成有关.
\end{abstract}

关键词：离子液体；二元混合；电导率；离子缔合；VTF方程

中图分类号: $\mathrm{O} 642$

\section{Ionic Association in Binary lonic Liquids by Conductivity}

\author{
NING Hui HOU Min-Qiang YANG De-Zhong KANG Xin-Chen HAN Bu-Xing* \\ (Institute of Chemistry, Chinese Academy of Sciences, CAS Key Laboratory of Colloid, Interface and Chemical Thermodynamics, \\ Beijing National Laboratory for Molecular Sciences, Beijing 100190, P. R. China)
}

\begin{abstract}
In this study, the conductivity of 13 ionic liquids (ILs) and 25 related binary mixtures were determined at temperatures ranging from $293.15-323.15 \mathrm{~K}$. The conductivity data of the pure ILs and their mixtures were fitted using the Vogel-Tammann-Fulcher (VTF) equation. The ionic association in the ILs and IL mixtures was discussed using the parameters of the VTF equation. It was demonstrated that at the same temperature, the ILs with short side chain cations, low charge density anions, and weak hydrogen bonding force between cations and anions usually exhibited high conductivity. Anions had a more obvious effect on conductivity than cations. The ionic association in the mixtures was affected not only by the species but also by the composition of the mixture.
\end{abstract}

Key Words: Ionic liquid; Binary mixture; Conductivity; Ionic association; VTF equation

\section{1 引言}

离子液体是由阴阳离子组成, 在较低温度下呈 液态的盐. 作为一种新型介质和功能流体, 离子液 体在化学反应、 ${ }^{1,2}$ 萃取分离、 ${ }^{3}$ 材料制备、 ${ }^{4}$ 电化学 ${ }^{5}$ 等 领域有广阔的应用前景.

离子液体体系中分子间相互作用研究是其应 用的基础, 具有重要的科学意义. 研究表明, 离子液 体结构复杂, 离子液体体系中存在多种分子间相互
作用, 如静电力、氢键、范德华力等. ${ }^{6}$ 目前, 离子液体 及其与分子溶剂间相互作用与物理化学性质的研 究已有很多报道, ${ }^{7-13}$ 但对二元离子液体混合体系的 分子间相互作用, 特别是其离子缔合作用的研究报 道较少. ${ }^{14} \mathrm{Mele}$ 等 ${ }^{15}$ 用核磁共振的方法对混合离子液 体体系中的离子缔合作用研究表明, 离子间的缔合 作用是影响其宏观性质的主要因素之一. Pandey等 ${ }^{16}$ 用溶剂化探针的方法研究了二元离子液体混合前

Received: February 15, 2013; Revised: April 16, 2013; Published on Web: April 17, 2013.

"Corresponding author. Email: hanbx@iccas.ac.cn; Tel: +86-10-62562821.

The project was supported by the National Natural Science Foundation of China (20903109, 21073207).

国家自然科学基金(20903109, 21073207)资助项目

(C) Editorial office of Acta Physico-Chimica Sinica 
后其阴阳离子缔合的变化, 指出离子间的缔合作用 是产生非理想混合现象的原因. 吴益华等 ${ }^{17}$ 通过质 谱研究表明, 1-丁基-3-甲基咪唑六氟磷酸盐和 1-丁 基-3-甲基咪唑双三氟甲磺酰亚胺盐混合后阴阳离 子间缔合作用减弱. Annat 等 ${ }^{18}$ 从分子结构的角度研 究了一些混合离子液体物理化学性质的变化规律, 指出离子缔合作用是混合体系物理化学性质随组 成非线性变化的主要因素之一。

虽然人们在离子液体性质研究方面开展了大 量的工作, 并取得一些重要进展, 但由于离子液体 体系中存在多种相互作用, 并且各种作用间相互影 响, 存在协同作用等, 离子液体中各种相互作用与 宏观性质之间关系的研究将是一项长期的工作. 特 别是对离子液体混合后阴阳离子间缔合作用的研 究, 目前还缺乏有效的手段, 本文以二元离子液体 混合物为对象, 通过测定离子液体电导率的方法研 究混合前后离子间的缔合作用与离子液体的种类 和混合物的组成的关系.

\section{2 实验部分}

\section{1 实验试剂与仪器}

实验所用离子液体列于表 1 , 其中所有纯离子 液体均由中国科学院兰州化学物理研究所绿色化 学与催化中心提供, 其纯度 $\geq 99 \%$. 混合离子液体按
照称重法配制. 离子液体的水分、质量、电导率分别 用 Karl Fischer 水分测定仪 (751 GPD Titrino, Metrohm, 瑞士), AR224CN 电子天平(Ohas Instruments Co., Ltd., 上海) 和 FE30K 电导率仪 (Mettler-Toledo Instruments Co., Ltd., 瑞士)测定. 恒温水浴的温度用 YKKY A2 温控仪(北京长流科学仪器有限公司)控 制.

\section{2 电导率测定}

在实验之前, 所有离子液体及其混合物在 $70{ }^{\circ} \mathrm{C}$ 真空烘箱中干燥 $72 \mathrm{~h}$, 并通过 Karl Fischer 水分测定 仪测定其水含量 $<0.05 \%(w)$. 电极电导常数用不同 浓度的 $\mathrm{KCl}$ 水溶液校正. 测量时, 将 $10 \mathrm{~mL}$ 离子液体 样品密封于玻璃试管中, 将试管浸没到恒温水浴 中, 每次测量前恒温 $1 \mathrm{~h}$, 每个温度点测量 3 次, 然后 取平均值, 测量误差 $\leq 0.5 \%$.

\section{3 结果与讨论}

\section{1 纯离子液体的电导率}

所有纯离子液体的电导率列于表 1 .

从表 1 可以看出, 在不同温度下本文对 BMIM- $\mathrm{PF}_{6}$ 电导率的测定结果与文献值 ${ }^{19-22}$ 的一致 性很好, 这证明了本文测定方法的可靠性. 表中的 结果表明, 在同一温度下不同离子液体的电导率顺 序为 EMIM-DCA $>$ EMIM-TFSI $>$ EMIM-TFO $>$ BMIM-

表 1 纯离子液体的电导率 $(\boldsymbol{k})$ 及 VTF 方程拟合参数

Table 1 Conductivity ( $\kappa$ ) of neat ILs and the fitting parameters of VTF equation

\begin{tabular}{|c|c|c|c|c|c|c|c|c|c|c|}
\hline \multirow{2}{*}{ IL } & \multicolumn{7}{|c|}{$\kappa /\left(\mathrm{mS} \cdot \mathrm{cm}^{-1}\right)$} & \multicolumn{3}{|c|}{ VTF parameters } \\
\hline & $293.15 \mathrm{~K}$ & $298.15 \mathrm{~K}$ & $303.15 \mathrm{~K}$ & $308.15 \mathrm{~K}$ & $313.15 \mathrm{~K}$ & $318.15 \mathrm{~K}$ & $323.15 \mathrm{~K}$ & $\kappa_{0} /\left(\mathrm{mS} \cdot \mathrm{cm}^{-1}\right)$ & $B / \mathrm{K}$ & $T_{0} / \mathrm{K}$ \\
\hline EMIM-DCA & 23.60 & 27.20 & 31.00 & 35.10 & 39.40 & 43.90 & 48.70 & 1553 & 601 & 150 \\
\hline EMIM-TFSI & 7.60 & 8.97 & 10.47 & 12.06 & 13.76 & 15.58 & 17.56 & 728 & 610 & 159 \\
\hline EMIM-TFO & 7.16 & 8.50 & 9.97 & 11.57 & 13.30 & 15.14 & 17.16 & 992 & 688 & 154 \\
\hline BMIM-SCN & 5.42 & 6.80 & 8.38 & 10.13 & 12.10 & 14.25 & 16.66 & 1343 & 648 & 176 \\
\hline BMIM-BF 4 & 2.83 & 3.63 & 4.58 & 5.64 & 6.91 & 8.30 & 9.93 & 2731 & 925 & 158 \\
\hline 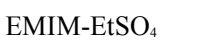 & 3.01 & 3.79 & 4.69 & 5.72 & 6.88 & 8.16 & 9.60 & 1236 & 757 & 167 \\
\hline BMIM-TFSI & 3.24 & 3.99 & 4.81 & 5.75 & 6.77 & 7.88 & 9.12 & 730 & 690 & 166 \\
\hline BPY-BF ${ }_{4}$ & 1.75 & 2.34 & 3.06 & 3.89 & 4.89 & 6.04 & 7.36 & 2049 & 823 & 175 \\
\hline $\mathrm{BMIM}-\mathrm{NO}_{3}$ & 1.06 & 1.47 & 1.99 & 2.61 & 3.37 & 4.26 & 5.31 & 2239 & 863 & 180 \\
\hline BMIM-PF $_{6}$ & 1.07 & 1.43 & 1.88 & 2.42 & 3.05 & 3.79 & 4.67 & 2478 & 990 & 165 \\
\hline BMIM-PF (lit.) & $1.09^{19}$ & $1.465^{20}$ & $1.96^{21}$ & $2.48^{20}$ & $2.97^{22}$ & $3.9^{20}$ & $4.77^{19}$ & - & - & - \\
\hline $\mathrm{CNPMIM} \mathrm{BF}_{4}$ & 0.411 & 0.601 & 0.861 & 1.19 & 1.60 & 2.11 & 2.73 & 2524 & 944 & 185 \\
\hline $\mathrm{BMIM}^{-\mathrm{HSO}_{4}}$ & 0.160 & 0.237 & 0.340 & 0.476 & 0.647 & 0.864 & 1.14 & 5224 & 1344 & 164 \\
\hline $\mathrm{HSO}_{3} \mathrm{BMIM}^{-\mathrm{TFO}}$ & 0.0641 & 0.0969 & 0.142 & 0.203 & 0.282 & 0.382 & 0.509 & 1888 & 1225 & 174 \\
\hline
\end{tabular}

EMIM: 1-ethyl-3-methylimidazolium, BMIM: 1-butyl-3-methylimidazolium, BPY: $N$-butylpyridinium,

CNPMIM: 1-cyanopropyl-3-methylimidazolium, $\mathrm{HSO}_{3} \mathrm{BMIM}$ 1-sulfobutyl-3-methylimidazolium, DCA: dicyanamide, EtSO 4 : ethylsulfate,

TFO: trifluoromethanesulfonate, TFSI: bis((trifluoromethyl)sulfonyl)imide, $\mathrm{BF}_{4}$ : tetrafluoroborate, $\mathrm{HSO}_{4}$ : hydrogen sulfate, $\mathrm{NO}_{3}$ : nitrate,

$\mathrm{PF}_{6}$ : hexafluorophosphate, $\mathrm{SCN}$ : thiocyanate; $\kappa_{0}, B, T_{0}$ are the fitting parameters. $\kappa_{0}$ means the conductivity of the ILs when the temperature is infinity; $T_{0}$ is associated with the melting point of the ILs, and the value is generally lower than the melting point of the ILs. 


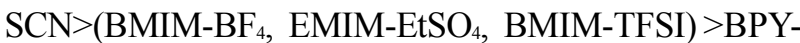
$\mathrm{BF}_{4}>\mathrm{BMIM}^{-\mathrm{NO}_{3}}>\mathrm{BMIM}-\mathrm{PF}_{6}>\mathrm{CN}-\mathrm{PMIM}-\mathrm{BF}_{4}>\mathrm{BMIM}-$ $\mathrm{HSO}_{4}>\mathrm{HSO}_{3} \mathrm{BMIM}-\mathrm{TFO}$, 所得结论与 $\mathrm{KunZ}^{\text {等 }}{ }^{20}$ 的结

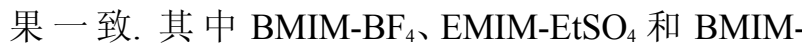
TFSI 的电导率差别较小, 其顺序与温度范围有关, 在 293.15-308.15 K 范围 内, BMIM-BF $<<$ EMIM-EtSO ${ }_{4}<$

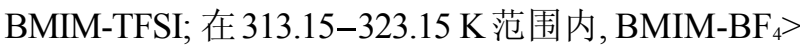
EMIM-EtSO ${ }_{4}>$ BMIM-TFSI.

Separovic 等 ${ }^{23}$ 指出, 虽然离子液体在结构上完 全由阴阳离子组成, 但是阴阳离子并不是完全解离 的, 它们在静电力、氢键、范德华力等的综合作用下 产生一定程度的离子缔合并形成离子对、离子网络 等结构, 并最终导致了离子液体的高粘度和低电导 率. Watanabe 等 ${ }^{21,24,25}$ 研究了阴阳离子的结构和缔合 对离子液体电导率的影响. 本文的结果与他们的结 论一致: 对于具有相同阴离子的离子液体, 阳离子 侧链越短, 电导率越大, 因为短链的阳离子具有较 小的范德华力, 因此缔合作用较小, ${ }^{26,27}$ 如: EMIMTFSI>BMIM-TFSI; 对于同样阳离子的离子液体, 阴 离子体积越小 (范德华力小), 电荷越分散(静电力 小), 与阳离子成氢键的作用越弱, 其缔合作用越弱, 电导率越大, ${ }^{28}$ 如: BMIM-SCN $>$ BMIM-TFSI $>$ BMIM$\mathrm{NO}_{3}>\mathrm{BMIM}^{-\mathrm{PF}_{6}}>\mathrm{BMIM}-\mathrm{HSO}_{4}$. 从这些结果还可以 看出, 电导率受阴离子的影响更显著. ${ }^{29}$ 如: 虽然阳 离子 BMIM 比 EMIM 的侧链多两个 $\mathrm{CH}_{2}$, 但电导率 顺序为: BMIM-SCN $>$ EMIM-EtSO $_{4}$.

离子液体的电导率 $(K)$ 受温度 $(T)$ 影响变化显著, 且随着温度的升高而增大(图 1), 其变化关系可以用 Vogel-Tammann-Fulcher (VTF)方程进行拟合:21

$$
\kappa=\kappa_{0} \exp \left(-B /\left(T-T_{0}\right)\right)
$$

其中 $\kappa_{0} 、 B 、 T_{0}$ 为拟合常数, 其数值列于表 $1 . \kappa_{0}$ 表示当 温度达到无穷大时离子液体的电导率; $T_{0}$ 与离子液 体的熔点有关, 其数值一般比离子液体的熔点低十 几到几十摄氏度.

VTF 方程是对阿伦尼乌斯公式(2)的修正:

$$
k=A \exp \left(-E_{\mathrm{a}} / R T\right)
$$

其中, $k$ 表示反应速率, $A$ 为指前因子, $E_{\mathrm{a}}$ 为表观活化 能, $R$ 为摩尔气体常数, $T$ 为反应温度. VTF 方程中的 参数 $B$ 与 $E_{\mathrm{a}}$ 具有类似的物理意义, ${ }^{29}$ 表示组成离子液 体的阴阳离子克服相互之间的缔合作用力而成为 自由导电离子所需能量的大小, ${ }^{30,31}$ 其值越大, 说明 产生离子液体缔合的作用力越强, 需要较高的能量 才能让离子克服相互之间的缔合作用力. ${ }^{32}$
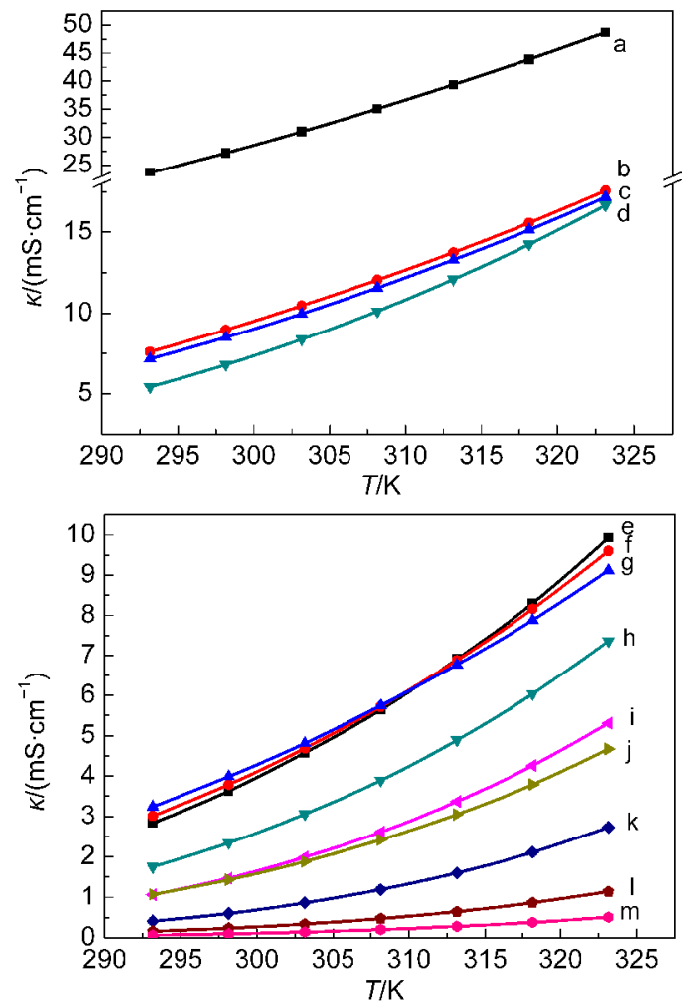

图 1 温度对纯离子液体电导率的影响

Fig.1 Dependence of conductivity of the neat ILs on temperature

(a) EMIM-DCA; (b) EMIM-TFSI; (c) EMIM-TFO; (d) BMIM-SCN (e) BMIM-BF ; (f) EMIM-EtSO ${ }_{4}$; (g) BMIM-TFSI; (h) BPY-BF (i) $\mathrm{BMIM}^{-\mathrm{NO}_{3}}$; (j) BMIM-PF ; (k) CNPMIM-BF ${ }_{4}$; (l) BMIM-HSO (m) $\mathrm{HSO}_{3} \mathrm{BMIM}-\mathrm{TFO}$

从表 1 中的数据可以看出, 离子液体的阳离子 侧链越长, 范德华力越大, 其 $B$ 值也越大, ${ }^{24}$ 如 BMIMTFSI>EMIM-TFSI; 阴离子与阳离子形成氢键的作 用力越强, 其 $B$ 值越大, ${ }^{21}$ 例如 $\mathrm{BMIM}-\mathrm{PF}_{6}>\mathrm{BMIM}-$ TFSI; 所以, $B$ 值的大小可以给出离子液体中阴阳离 子间缔合作用的强弱的信息.

\section{2 等摩尔混合离子液体体系的电导率}

为了研究离子液体混合后电导率的变化规律, 本文首先在 293.15-323.15 K 温度范围内测定了 15 组等摩尔混合离子液体体系的电导率(表 2). 研究表 明, 等摩尔混合离子液体的电导率都介于组成混合 离子液体的两种纯离子液体的电导率之间, 且随温 度升高而增大, 其变化关系符合 VTF 方程, 其拟合 常数列于表 2 , 拟合曲线如图 2 所示.

根据上面对 VTF 方程中参数 $B$ 的分析, 若离子 液体是理想混合溶液, 则可根据公式(3)计算二元离 子液体混合物的 $B$ 值:

$$
B_{\text {cal }}=x_{1} B_{1}+x_{2} B_{2}
$$


表 2 等摩尔混合离子液体的电导率及VTF 方程拟合参数

Table 2 Conductivity of equal molar binary mixtures of ILs and the fitting parameters of VTF equation

\begin{tabular}{|c|c|c|c|c|c|c|c|c|c|c|c|c|}
\hline \multirow{2}{*}{ IL mixture } & \multicolumn{7}{|c|}{$\kappa /\left(\mathrm{mS} \cdot \mathrm{cm}^{-1}\right)$} & \multicolumn{5}{|c|}{ VTF parameters } \\
\hline & $293.15 \mathrm{~K}$ & $298.15 \mathrm{~K}$ & $303.15 \mathrm{~K}$ & $308.15 \mathrm{~K}$ & $313.15 \mathrm{~K}$ & $318.15 \mathrm{~K}$ & $323.15 \mathrm{~K}$ & $\kappa_{0} /\left(\mathrm{mS} \cdot \mathrm{cm}^{-1}\right)$ & $B_{\text {exp }} / \mathrm{K}$ & $B_{\text {cal }} / \mathrm{K}$ & $E$ & $T_{0} / \mathrm{K}$ \\
\hline EMIM-DCA+BMIM-SCN & 12.63 & 15.09 & 17.76 & 20.70 & 23.80 & 27.10 & 30.70 & 1119 & 546 & 624 & -12.5 & 171 \\
\hline EMIM-DCA+EMIM-TFSI & 11.22 & 13.13 & 15.20 & 17.41 & 19.76 & 22.30 & 25.00 & 1126 & 658 & 606 & 8.6 & 150 \\
\hline EMIM-DCA+EMIM-EtSO & 8.69 & 10.48 & 12.43 & 14.57 & 16.88 & 19.37 & 22.10 & 1164 & 627 & 679 & -7.7 & 165 \\
\hline EMIM-TFO+EMIM-TFSI & 7.03 & 8.34 & 9.77 & 11.31 & 12.97 & 14.72 & 16.63 & 678 & 591 & 649 & -8.9 & 164 \\
\hline EMIM-TFO+BMIM-SCN & 6.10 & 7.44 & 8.92 & 10.58 & 12.39 & 14.34 & 16.50 & 1127 & 666 & 668 & -0.3 & 166 \\
\hline EMIM-TFSI+BMIM-SCN & 5.96 & 7.18 & 8.57 & 10.08 & 11.73 & 13.50 & 15.41 & 807 & 613 & 629 & -2.5 & 168 \\
\hline $\mathrm{EMIM} \mathrm{EtSO}_{4}+\mathrm{BMIM}-\mathrm{SCN}$ & 3.68 & 4.65 & 5.75 & 7.02 & 8.45 & 10.02 & 11.79 & 1396 & 731 & 702 & 4.1 & 170 \\
\hline EMIM-EtSO $_{4}+$ EMIM-TFSI & 4.09 & 4.99 & 6.03 & 7.15 & 8.41 & 9.76 & 11.25 & 804 & 668 & 684 & -2.3 & 167 \\
\hline EMIM-TFSI+BMIM-PF 6 & 3.54 & 4.36 & 5.29 & 6.34 & 7.50 & 8.77 & 10.18 & 1041 & 748 & 800 & -6.5 & 162 \\
\hline BMIM-NO ${ }_{3}+\mathrm{BMIM}-\mathrm{SCN}$ & 2.42 & 3.17 & 4.06 & 5.11 & 6.33 & 7.71 & 9.30 & 2177 & 828 & 756 & 9.5 & 171 \\
\hline BMIM-SCN+BMIM-PF 6 & 2.59 & 3.34 & 4.21 & 5.23 & 6.40 & 7.72 & 9.22 & 1953 & 839 & 819 & 2.4 & 166 \\
\hline $\mathrm{BMIM}-\mathrm{BF}_{4}+\mathrm{BPY}-\mathrm{BF}_{4}$ & 2.23 & 2.92 & 3.74 & 4.68 & 5.82 & 7.08 & 8.55 & 2476 & 889 & 874 & 1.7 & 166 \\
\hline EMIM-TFSI+CN-PMIM-BF & 2.35 & 3.00 & 3.75 & 4.62 & 5.58 & 6.68 & 7.91 & 1093 & 750 & 777 & -3.5 & 171 \\
\hline EMIM-TFSI+BMIM-HSO & 1.78 & 2.27 & 2.83 & 3.48 & 4.20 & 5.02 & 5.96 & 1161 & 852 & 977 & -12.8 & 162 \\
\hline EMIM-TFSI+HSO ${ }_{3} \mathrm{BMIM}-\mathrm{TFO}$ & 0.939 & 1.23 & 1.58 & 1.99 & 2.46 & 3.00 & 3.62 & 868 & 834 & 918 & -9.2 & 171 \\
\hline
\end{tabular}

$B_{\text {exp. }}$ and $B_{\text {cal }}$ are calculated from equations (1) and (3), respectively; $E$ is the relative deviation between $B_{\text {exp. }}$ and $B_{\text {cal }}$ in percent.
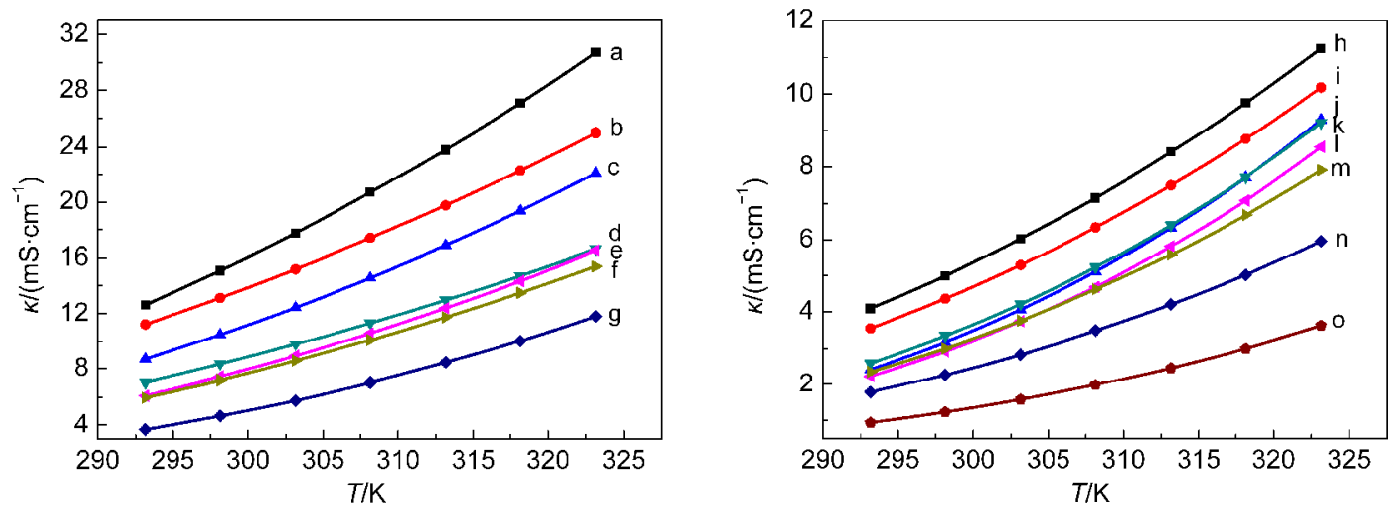

图 2 温度对等摩尔离子液体混合物电导率的影响

Fig.2 Dependence of conductivity of the equal mole binary mixtures of ILs on temperature

(a) EMIM-DCA+BMIM-SCN; (b) EMIM-DCA+EMIM-TFSI; (c) EMIM-DCA+EMIM-EtSO ; (d) EMIM-TFO+EMIM-TFSI;

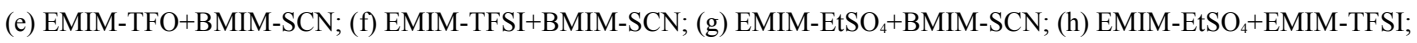

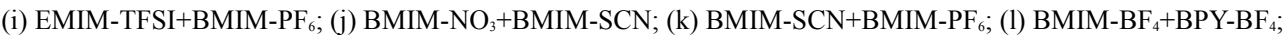

(m) EMIM-TFSI+CN-PMIM-BF $;$; (n) EMIM-TFSI+BMIM-HSO 4 ; (o) EMIM-TFSI+HSO ${ }_{3} \mathrm{BMIM-TFO}$

$B_{2}$ 对应纯离子液体的 $B$ 值.

对于非理想混合的情况, 实验值 $B_{\text {exp. }}$ 与计算值

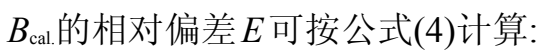

$$
E=\left(B_{\text {exp. }}-B_{\text {cal. }}\right) / B_{\text {cal. }} \times 100 \%
$$

若 $E$ 为正值, 说明离子液体混合后的阴阳离子 成为导电的自由离子需要的能量相对增大, 离子间 的缔合作用增强; 若 $E$ 为负值, 说明阴阳离子间缔合 作用减弱.

MacFarlane 等 ${ }^{33}$ 发现当 EMIM-TFO 和 EMIMTFSI 混合后, 阴阳离子缔合作用减弱, 导电性增强, 同样的效应也存在于 EMIM-BF $4+\mathrm{EMIM}_{4} \mathrm{DCA}$ 体系 中. ${ }^{34}$ 本文根据离子液体混合前后 VTF 方程中参数 $B$
的相对偏差 $E$ 分析了二元离子液体混合物中的离子 缔合作用, 发现 EMIM-TFO+EMIM-TFSI 等摩尔混 合后, 其 $E$ 值为 -8.9 , 说明 TFO-TFSI 混合减弱了离 子间的缔合作用, 得到了与 MacFarlane 等一致的规 律, 证明了本文所用方法的合理性.

同时, 从表 2 所示的 15 组混合离子液体的 $E$ 值 可以看出, 对于同一阳离子EMIM, 等摩尔混合后离 子液体 TFO-TFSI、DCA-EtSO ${ }_{4}$ 和 $\mathrm{EtSO}_{4}-\mathrm{TFSI}$ 中阴阳 离子间的缔合作用减弱, $E$ 表现为负值; 而 DCATFSI 表现出较强的缔合增强效应, 其 $E$ 为正值; 对 于同一阳离子 $\mathrm{BMIM}$, 等摩尔混合后离子液体 $\mathrm{NO}_{3}$ $\mathrm{SCN}$ 和 $\mathrm{SCN}-\mathrm{PF}_{6}$ 体系中阴阳离子间的缔合作用增 
强, $E$ 表现为正值; 对于同一阴离子 $\mathrm{BF}_{4}, \mathrm{BMIM-BPY}$ 呈现缔合增强效应, 其 $E$ 值略大于零.

若参与混合的离子液体的阴阳离子都不相同, 其 $E$ 值的变化规律比较复杂. 如 BMIM-TFSI + BMIM-SCN 体系表现出较强的缔合增强效应, 其 $E$ 值为 9.3 (表 3), 而 EMIM-TFSI+BMIM-SCN 表现出 缔合减弱效应, 其 $E$ 值为 -2.5 , 这说明 EMIM-BMIM 呈现明显的缔合减弱效应, 超过了 TFSI-SCN 之间 的缔合增强效应, 所以在本文所研究的体系中, 大 多数 EMIM-BMIM 混合体系的 $E$ 都为负值, 如: EMIM-TFSI+BMIM-HSO 4 , EMIM-DCA+BMIM-SCN, EMIM-TFSI+BMIM-PF 6 , EMIM-TFSI+BMIM-SCN 和 EMIM-TFO+BMIM-SCN, 但是 EMIM-EtSO ${ }_{4}+\mathrm{BMIM}^{-}$ $\mathrm{SCN}$ 体系除外, 其 $E$ 值为 4.1. 这说明阴离子 $\mathrm{EtSO}_{4}-$ SCN 之间具有较强的缔合增强效应, 超过了 EMIM-BMIM 的缔合减弱效应. 值得注意的是, EMIM-TFO+BMIM-SCN 的 $E$ 值虽然为负, 但几乎
等于零, 可以认为 EMIM-BMIM 的缔合减弱效应与 TFO-SCN 的缔合增强效应相当，二者混合后基本符 合理想混合规律. 因此, 混合离子液体中的缔合作 用远比纯离子液体复杂得多, 特别是对于阴阳离子 都不同的离子液体混合情况, 其中阳离子和阴离子 各自起作用的程度还有待进一步研究.

\section{3 全浓度范围混合离子液体的电导率}

为了更详细地研究二元离子液体混合体系中 离子间的缔合作用, 本文还研究了 3 组全浓度范围 内混合离子液体的 $E$ 值随组成的变化规律. 其中, BMIM-TFSI+BMIM-SCN 和 BMIM-PF 6 - BMIM-BF $_{4}$ 的电导率和 VTF 方程拟合参数列于表 3 , 拟合曲线 如图 3 所示. 图 4 给出了 3 个二元系的 $E$ 值随组成的 变化, 其中 $\mathrm{HMIM}-\mathrm{PF}_{6}+\mathrm{HMIM}-\mathrm{Cl}$ 体系的 $E$ 值用我们 以前报道的电导率数据 ${ }^{35}$ 计算得到.

从图 4 可以看出, 对于体系 BMIM-TFSI+BMIM$\mathrm{SCN}$, 其 $E$ 值在整个混合浓度范围内都是正值, 说明

表 3 不同浓度混合离子液体的电导率及 VTF 方程拟合参数

Table 3 Conductivity of binary IL mixtures in the whole concentration range and the fitting parameters of VTF equation

\begin{tabular}{|c|c|c|c|c|c|c|c|c|c|c|c|c|}
\hline \multirow{2}{*}{$x_{2}$} & \multicolumn{7}{|c|}{$\kappa /\left(\mathrm{mS} \cdot \mathrm{cm}^{-1}\right)$} & \multicolumn{5}{|c|}{ VTF parameters } \\
\hline & $293.15 \mathrm{~K}$ & $298.15 \mathrm{~K}$ & $303.15 \mathrm{~K}$ & $308.15 \mathrm{~K}$ & $313.15 \mathrm{~K}$ & $318.15 \mathrm{~K}$ & $323.15 \mathrm{~K}$ & $\kappa_{0} /\left(\mathrm{mS} \cdot \mathrm{cm}^{-1}\right)$ & $B_{\text {exp }} / \mathrm{K}$ & $B_{\text {cal }} / \mathrm{K}$ & $E$ & $T_{0} / \mathrm{K}$ \\
\hline \multicolumn{13}{|c|}{ BMIM-TFSI(1)+BMIM-SCN(2) } \\
\hline 0.2 & 3.27 & 4.03 & 4.88 & 5.83 & 6.89 & 8.03 & 9.30 & 774 & 695 & 682 & 1.9 & 166 \\
\hline 0.4 & 3.45 & 4.25 & 5.16 & 6.18 & 7.32 & 8.56 & 9.93 & 972 & 734 & 673 & 9.1 & 163 \\
\hline 0.5 & 3.62 & 4.48 & 5.43 & 6.51 & 7.71 & 9.01 & 10.47 & 1019 & 731 & 669 & 9.3 & 164 \\
\hline 0.6 & 3.79 & 4.70 & 5.73 & 6.90 & 8.18 & 9.58 & 11.17 & 1088 & 721 & 665 & 8.4 & 166 \\
\hline 0.86 & 4.69 & 5.83 & 7.15 & 8.62 & 10.27 & 12.06 & 14.07 & 1297 & 695 & 654 & 6.3 & 170 \\
\hline \multicolumn{13}{|c|}{ 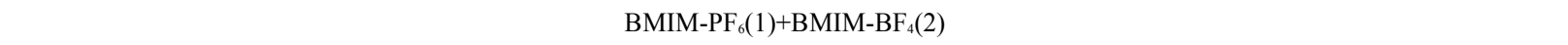 } \\
\hline 0.1 & 1.25 & 1.65 & 2.15 & 2.74 & 3.46 & 4.27 & 5.23 & 3025 & 1037 & 984 & 5.4 & 160 \\
\hline 0.3 & 1.48 & 1.95 & 2.53 & 3.19 & 4.01 & 4.92 & 5.99 & 2782 & 995 & 971 & 2.5 & 161 \\
\hline 0.5 & 1.78 & 2.34 & 3.01 & 3.77 & 4.69 & 5.74 & 6.95 & 2792 & 975 & 957 & 1.9 & 161 \\
\hline 0.7 & 2.13 & 2.77 & 3.53 & 4.39 & 5.44 & 6.61 & 7.95 & 2591 & 938 & 945 & -0.7 & 161 \\
\hline 0.9 & 2.58 & 3.32 & 4.20 & 5.19 & 6.38 & 7.71 & 9.21 & 2452 & 903 & 932 & -3.1 & 161 \\
\hline
\end{tabular}
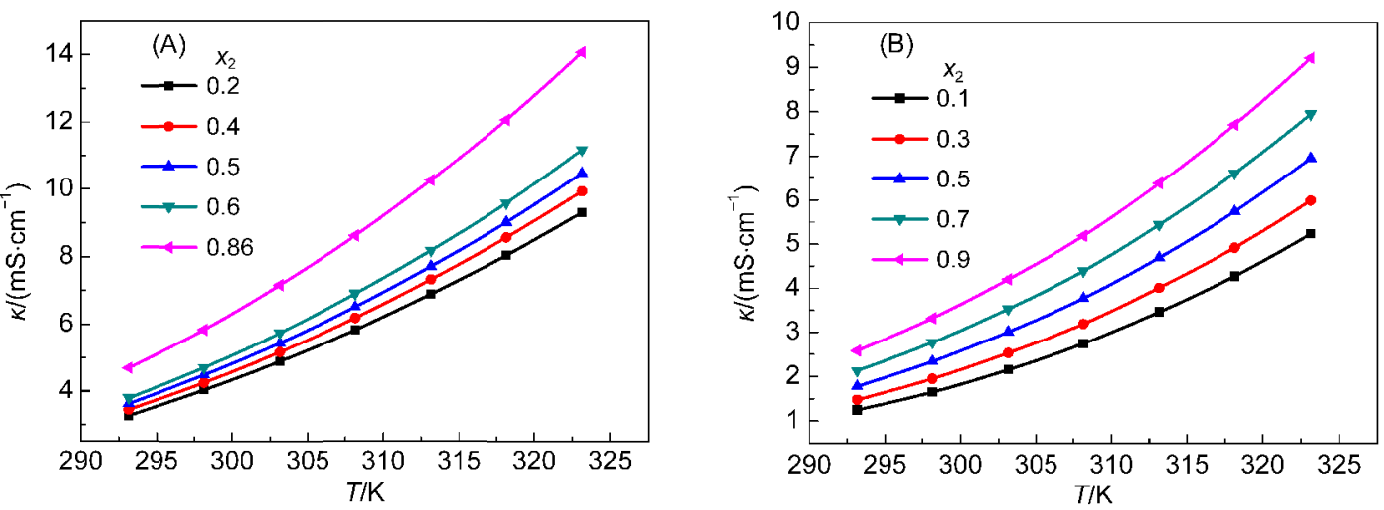

图 3 温度对不同浓度离子液体混合物电导率的影响

Fig.3 Dependence of conductivity of the IL mixtures on temperature

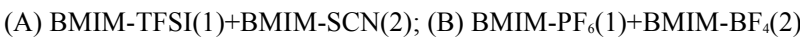




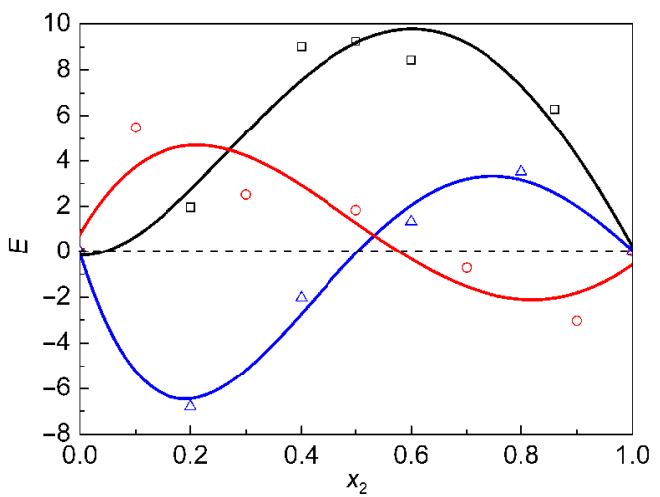

图 4 离子液体混合物的组成对 $E$ 的影响

Fig.4 Dependence of $E$ on the mole fraction of the binary IL mixtures

( ) BMIM-TFSI+BMIM-SCN, ( ( ) BMIM-PF + BMIM-BF $_{4}$, $(\triangle)$ HMIM-PF 6 +HMIM-Cl

无论 BMIM-TFSI 与 BMIM-SCN 以何种比例混合, 混合后阴阳离子间的缔合作用都增强, 但增强的程 度与组成有关, 在摩尔比为 $1: 1$ 时达到最大. 体系

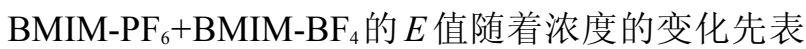
现为正值, 然后为负值, 说明在富 BMIM-PF。 区域, 混合后阴阳离子缔合作用增强, 而在富 $\mathrm{BMIM}^{-\mathrm{BF}_{4}}$ 区域, 混合后阴阳离子缔合作用减弱. 与此相反的 是, $\mathrm{HMIM}-\mathrm{PF}_{6}+\mathrm{HMIM}-\mathrm{Cl}$ 体系的 $E$ 先表现为负值, 然后为正值, 说明离子缔合作用随着 HMIM-Cl 浓度 的增加先减弱后增强. 这些现象说明, 离子液体混 合后阴阳子间缔合作用的变化不仅与混合体系中 离子液体的种类相关, 还与其组成有关.

\section{4 结 论}

本文对一系列离子液体及其混合物的电导率 和离子间的缔合作用进行了研究. 结果表明, 离子 液体的电导率与其阴阳离子组成有关, 阳离子侧链 越短, 阴离子电荷越分散, 阴阳离子间的氢键作用 力越弱, 缔合作用越小, 离子液体的电导率越大, 其 中阴离子的影响相对阳离子更明显. 无论是纯离子 液体还是其混合物, 电导率随温度的变化都符合 VTF 方程, 且混合离子液体的电导率介于两种参与 混合的纯离子液体的电导率之间. 对 VTF 方程中参 数 $B$ 的分析表明, 离子液体混合后离子液体间缔合 作用的增强或减弱不仅取决于离子液体的种类, 还 取决于其混合物的组成. 这些结果可为通过离子液 体混合的方法调控离子液体的性质提供参考.

\section{References}

(1) Gong, Y. Y.; Liu, M.; Jia, S. Y.; Feng, J. P.; Song, C. S.; Guo, X.
W. Acta Phys. -Chim. Sin. 2012, 28, 686. [公艳艳, 刘 民, 贾 松岩, 冯建萍, 宋春山, 郭新闻. 物理化学学报, 2012, 28, 686.] doi: 10.3866/PKU.WHXB201112292

(2) Yuan, X.; Lou, S. J.; Kou, Y. Acta Phys. -Chim. Sin. 2010, 26, 908. [苑 晓, 娄舒洁, 寇 元. 物理化学学报, 2010, 26, 908.] doi: 10.3866/PKU.WHXB20100433

(3) Chen, Y.; Han, J.; Wang, T.; Mu, T. C. Energy \& Fuels 2011, 25 , 5810. doi: $10.1021 / \mathrm{ef} 201519 \mathrm{~g}$

(4) Zhao, X. Y.; Cao, Y. R.; Cao, G. R.; Xiao, R. J. Acta Phys. -Chim. Sin. 2012, 28, 1411. [赵学艳, 曹宇容, 曹桂荣, 肖瑞杰. 物理化学学报, 2012, 28, 1411.] doi: 10.3866/PKU. WHXB201203262

(5) Zhong, H. X.; Zhao, C. B.; Luo, H.; Zhang, L. Z. Acta Phys. -Chim. Sin. 2012, 28, 2641. [仲皓想, 赵春宝, 骆 浩, 张灵志. 物理化学学报, 2012, 28, 2641.] doi: 10.3866/PKU. WHXB201207181

(6) Dong, K.; Song, Y. T.; Liu, X. M.; Cheng, W. G.; Yao, X. Q.; Zhang, S. J. J. Phys. Chem. B 2012, 116, 1007. doi: 10.1021/ jp205435u

(7) Xu, W. G.; Li, L.; Ma, X. X.; Wei, J.; Duan, W. B.; Guan, W.; Yang, J. Z. J. Chem. Eng. Data 2012, 57, 2177. doi: 10.1021/ je3000348

(8) Zhang, Q. G.; Wei, Y.; Sun, S. S.; Wang, C.; Yang, M.; Liu, Q. S.; Gao, Y. A. J. Chem. Eng. Data 2012, 57, 2185. doi: 10.1021/ je300153f

(9) Wang, D.; Tian, G. C. Acta Phys. -Chim. Sin. 2012, 28, 2558. [王 丁, 田国才. 物理化学学报, 2012, 28, 2558.] doi: 10.3866/ PKU.WHXB201208271

(10) Ren, S. H.; Hou, Y. C.; Wu, W. Z.; Liu, Q. Y.; Xiao, Y. F.; Chen, X. T. J. Phys. Chem. B 2010, 114, 2175. doi: 10.1021/jp9108859

(11) Zhang, L. Q.; Li, H. R. Acta Phys. -Chim. Sin. 2010, 26, 2877. [张力群, 李浩然. 物理化学学报, 2010, 26, 2877.] doi: 10.3866/ PKU.WHXB20101123

(12) Zhai, C. P.; Liu, X. J.; Zhao, Y.; Wang, J. J. Acta Phys. -Chim. Sin. 2009, 25, 1185. [翟翠萍, 刘学军, 赵 扬, 王键吉. 物理 化学学报, 2009, 25, 1185.] doi: 10.3866/PKU.WHXB20090628

(13) Zhang, Q. G.; Wang, N. N.; Yu, Z. W. J. Phys. Chem. B 2010, 114, 4747. doi: $10.1021 /$ jp 1009498

(14) Niedermeyer, H.; Hallett, J. P.; Villar-Garcia, I. J.; Hunt, P. A.; Welton, T. Chem. Soc. Rev. 2012, 41, 7780. doi: 10.1039/ c2 $\operatorname{cs} 35177 \mathrm{c}$

(15) Castiglione, F.; Raos, G.; Battista Appetecchi, G.; Montanino, M.; Passerini, S.; Moreno, M.; Famulari, A.; Mele, A. Phys. Chem. Chem. Phys. 2010, 12, 1784. doi: 10.1039/b921816e

(16) Fletcher, K. A.; Baker, S. N.; Baker, G. A.; Pandey, S. New J. Chem. 2003, 27, 1706. doi: 10.1039/b305965k

(17) Ma, L. L.; Liu, Y. F.; Yuan, J.; Wu, Y. H. Chemical Journal of Chinese Universities 2006, 27, 2182. [马亮亮, 刘逸枫, 袁 俊, 吴益华. 高等学校化学学报, 2006, 27, 2182.]

(18) Annat, G.; Forsyth, M.; MacFarlane, D. R. J. Phys. Chem. B 2012, 116, 8251. doi: 10.1021/jp3012602 
(19) Widegren, J. A.; Saurer, E. M.; Marsh, K. N.; Magee, J. W. J. Chem. Thermodyn. 2005, 37, 569. doi: 10.1016/j.jct. 2005.04.009

(20) Zech, O.; Stoppa, A.; Buchner, R.; Kunz, W. J. Chem. Eng. Data 2010, 55, 1774. doi: 10.1021/je900793r

(21) Tokuda, H.; Hayamizu, K.; Ishii, K.; Abu Bin Hasan Susan, M.; Watanabe, M. J. Phys. Chem. B 2004, 108, 16593. doi: 10.1021/ jp047480r

(22) Zhang, J. M.; Yang, C. H.; Hou, Z. S.; Han, B. X.; Jiang, T.; Li, X. H.; Zhao, G. Y.; Li, Y. F.; Liu, Z. M.; Zhao, D. B.; Kou, Y. New J. Chem. 2003, 27, 333. doi: 10.1039/b206526f

(23) Burrell, G. L.; Burgar, I. M.; Gong, Q.; Dunlop, N. F.; Separovic, F. J. Phys. Chem. B 2010, 114, 11436.

(24) Tokuda, H.; Hayamizu, K.; Ishii, K.; Susan, M.; Watanabe, M. J. Phys. Chem. B 2005, 109, 6103. doi: 10.1021/jp044626d

(25) Tokuda, H.; Ishii, K.; Susan, M.; Tsuzuki, S.; Hayamizu, K.; Watanabe, M. J. Phys. Chem. B 2006, 110, 2833. doi: 10.1021/ jp053396f

(26) Hagiwara, R.; Matsumoto, K.; Nakamori, Y.; Tsuda, T.; Ito, Y.; Matsumoto, H.; Momota, K. J. Electrochem. Soc. 2003, 150, D195.

(27) Yoshida, Y.; Baba, O.; Saito, G. J. Phys. Chem. B 2007, 111, 4742. doi: 10.1021/jp067055t

(28) Leys, J.; Rajesh, R. N.; Menon, P. C.; Glorieux, C.; Longuemart,
S.; Nockemann, P.; Pellens, M.; Binnemans, K. J. Chem. Phys. 2010, 133, 034503. doi: 10.1063/1.3455892

(29) Bandres, I.; Montano, D. F.; Gascon, I.; Cea, P.; Lafuente, C. Electrochim. Acta 2010, 55, 2252. doi: 10.1016/j.electacta. 2009.11.073

(30) Liu, Q. S.; Yan, P. F.; Yang, M.; Tan, Z. C.; Li, C. P.; WelzBiermann, U. Acta Phys. -Chim. Sin. 2011, 27, 2762. [刘青山, 颜佩芳, 杨 沝, 谭志诚, 李长平, Welz-Biermann, U. 物理化学 学报, 2011, 27, 2762.] doi: 10.3866/PKU.WHXB20112762

(31) Noda, A.; Hayamizu, K.; Watanabe, M. J. Phys. Chem. B 2001, 105, 4603. doi: 10.1021/jp004132q

(32) Leys, J.; Wubbenhorst, M.; Menon, C. P.; Rajesh, R.; Thoen, J.; Glorieux, C.; Nockemann, P.; Thijs, B.; Binnemans, K.; Longuemart, S. J. Chem. Phys. 2008, 128, 64509. doi: 10.1063/ 1.2827462

(33) Every, H.; Bishop, A. G.; Forsyth, M.; MacFarlane, D. R. Electrochim. Acta 2000, 45, 1279. doi: 10.1016/S0013-4686(99) 00332-1

(34) Stoppa, A.; Buchner, R.; Hefter, G. J. Mol. Liq. 2010, 153, 46.

(35) Ning, H.; Hou, M. Q.; Mei, Q. Q.; Liu, Y. H.; Yang, D. Z.; Han, B. X. Science China Chemistry 2012, 55, 1509. ［宁＼cjkstart汇，侯民 强, 梅清清, 刘元会, 杨德重, 韩布兴. 中国科学: 化学, 2012, 55, 1509.] doi: 10.1007/s11426-012-4655-1 